\title{
Odds Ratio: Reflexão sobre a Validade de uma Medida de Referência em Epidemiologia
}

\author{
Odds Ratio: Review about the Meaning of an Epidemiological Measure
}

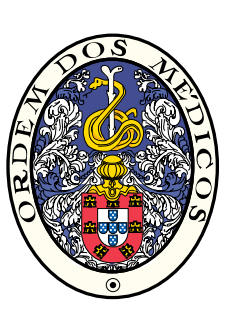

\author{
Pedro AGUIAR ${ }^{1}$, Baltazar NUNES ${ }^{2}$ \\ Acta Med Port 2013 Sep-Oct;26(5):505-510
}

RESUMO

Introdução: É importante rever a validade do Odds Ratio como medida de associação e efeito, assim como, qual o viés introduzido pelo Odds Ratio quando este representa uma razão de riscos ou uma razão de prevalências em situação de doença mais frequente. Material e Métodos: Simulámos numa coorte de 200 indivíduos com 100 expostos e 100 não expostos a um fator de risco, um cenário de doença rara e outro de doença mais frequente, com razão de riscos igual em ambos os cenários. Determinaram-se o Odds Ratio e o Risco Relativo pelo método clássico (padrão) e respectivamente por regressão logística e regressão de Poisson. Introduziu-se de seguida uma variável de confundimento e determinaram-se o Odds Ratio e o Risco Relativo pelo método de Mantel-Hanszel (análise estratificada padrão) e respectivamente por regressão logística e regressão de Poisson. As análises estatísticas foram efectuadas em SPSS V20.

Resultados: Para a doença rara, o Odds Ratio aproximou-se do Risco Relativo. Quando a doença foi mais frequente, o Odds Ratio sobrestimou o Risco Relativo. Nesta situação, e com a presença de uma variável de confundimento, o Risco Relativo ajustado por regressão de Poisson permitiu obter estimativas mais válidas da razão de riscos que o Odds Ratio ajustado por regressão logística. Os intervalos de confiança do Risco Relativo estimado por regressão de Poisson foram sempre mais largos que os determinados por análise de Mantel-Hanszel.

Conclusões: O Odds Ratio e a regressão logística múltipla são procedimentos válidos em estudos caso-controlo e em estudos prospetivos e transversais de natureza exploratória. O Odds Ratio não deve ser interpretado como uma razão de riscos ou razão de prevalências se o resultado de saúde não é raro. A análise de regressão múltipla de Poisson deve ser considerada como alternativa válida à regressão logística múltipla, especialmente em estudos de uma exposição específica.

Palavras-chave: Análise de Regressão; Desenho de Investigações Epidemiológicas; Distribuição de Poisson; Razão de Chances; Modelos Logísticos; Medição do Risco.

\section{ABSTRACT}

Introduction: It is very important to review the meaning of the Odds Ratio as a measure of effect and association, as well as, the bias of the Odds Ratio when it is assumed as a risk ratio or a prevalence ratio in the case of frequent disease or frequent health outcome. Material and Methods: We simulated in a cohort of 200 individuals with 100 exposed and 100 non-exposed to a risk factor, a first setting of rare disease and a second setting of a more frequent disease. In both settings the risk ratios were similar. We computed the Odds Ratio and Relative Risks by the classical approach (standard method) and respectively by logistic regression and Poisson regression. After these, we introduced in the cohort a confounding variable and then we computed the Odds Ratio and Relative Risk by Mantel-Hanszel stratified analysis (standard method) and respectively by multiple logistic regression and multiple Poisson regression. We used the $95 \%$ confidence interval in parameter estimation and SPSS V20 was used in statistical analysis.

Results: In the case of rare disease the Odds Ratio was very close to the Relative Risk. For more frequent disease the Odds Ratio overestimated the Relative Risk. In this situation and with a confounding variable, the relative Risk adjusted by Poisson regression was more valid then the Odds Ratio to represent a risk ratio. The confidence intervals of the Relative Risk adjusted by Poisson regression were always greater than Mantel-Hanszel confidence intervals.

Conclusions: The Odds Ratio and multiple logistic regression were valid analytic procedures in several epidemiological designs such as case-control studies and exploratory prospective studies as well as exploratory cross-sectional studies. The Odds Ratio should not be interpreted as a risk ratio or a prevalence ratio in the case of a health outcome that it is not rare. The multiple Poisson regression should be considered as an alternative procedure to logistic regression, especially if we want to estimate the effect of a specific exposure to a risk factor.

Keywords: Odds Ratio; Logistic Models; Epidemiologic Research Design; Risk Assessment; Poisson Distribution; Regression Analysis.

\section{INTRODUÇÃO}

Em 1961 McBride escreve uma carta à Lancet, a questionar se a comunidade médica tinha notado algum aumento da incidência de malformação congénita, em recém-nascidos de mães que tinham usado Talidomida durante a gravidez. ${ }^{1}$ No seguimento desta carta, Mellin e Katzenstein ${ }^{2}$ referem a observação de 41 malformações em 46 grávidas que tomaram Talidomida durante a gravidez, em compara- ção com 300 grávidas sem qualquer caso de malformação e que não tomaram Talidomida (Tabela 1).

Com estes dados, facilmente determinamos que a possibilidade de malformação congénita para as mães expostas à Talidomida seria de 41 para 5 , ou seja, $41 / 5=8,2$. Este resultado, não é nada mais nada menos que o odds de malformação para as mães expostas à Talidomida, indicando

1. Departamento de Epidemiologia e Estatística. Escola Nacional de Saúde Pública/Universidade Nova de Lisboa. Lisboa. Portugal.

2. Departamento de Epidemiologia. Instituto Nacional de Saúde Dr. Ricardo Jorge. Lisboa. Portugal.

Recebido: 21 de Junho de 2013 - Aceite: 10 de Julho de 2013 | Copyright @ Ordem dos Médicos 2013 
Tabela 1 - Associação entre malformação congénita do feto e uso de talidomida pela mãe, adaptado de Mellin e Katzenstein (1962)²

Malformação congénita do feto

\begin{tabular}{lcc}
\hline Uso de talidomida & Sim & Não \\
Sim & 41 & 5 \\
Não & 0 & 300
\end{tabular}

que existe uma razão de probabilidades de malformação de 8 para 1 para as mães expostas à Talidomida. Admitindo numa prespectiva mais conservadora, que mesmo sem a toma de Talidomida seria possível o aparecimento de um caso de malformação, então o odds de malformação para as mães não expostas à Talidomida seria de 1 para 300 , ou seja, $1 / 300=0,003$. Este resultado indicaria que a possibilidade de malformação para as mães não expostas à Talidomida seria próximo de 0 . Com estes dois resultados facilmente poderíamos determinar a razão dos dois odds o odds ratio $(\mathrm{OR})$ - ou seja, OR $=(41 / 5) /(1 / 300)=2460$. Fazendo uma possível interpretação deste OR como razão de riscos de malformação entre expostos e não expostos à Talidomida, seria sugerido que o risco de malformação congénita era 2460 vezes, caso a grávida tivesse consumido Talidomida durante a gravidez. Para termos uma ideia da grandeza desta estimativa catastrófica de risco relativo da exposição à Talidomida, basta compararmos com o risco relativo de morte em consequência da exposição ao fumo de tabaco, que é avaliado em três vezes no estudo de Doll. ${ }^{3}$

O OR é uma das medidas de associação e efeito de utilização mais comum na investigação epidemiológica, podendo a sua definição ser facilmente encontrada em qualquer livro de referência em Epidemiologia tal como o Dicionário de Epidemiologia de Last, ${ }^{4}$ o Modern Epidemiology de Rothman e Greenland, ${ }^{5}$ ou no livro mais prático de Epidemiologia de Gordis. ${ }^{6}$ Em termos específicos o OR é uma razão entre duas possibilidades (odds). O odds da ocorrência da doença é diferente da probabilidade ou risco da ocorrência da doença. Enquanto que a probabilidade de ocorrência da doença é dada pelo número de casos da doença a dividir pelo total de indivíduos em risco, o odds é dado pela razão entre a probabilidade da doença ocorrer e a probabilidade da doença não ocorrer, o que resulta no número de casos da doença a dividir pelo número de indivíduos que não desenvolveram a doença. A aplicação prática do OR como medida de associação ou efeito também pode ser facilmente encontrada na literatura de investigação epidemiológica, por exemplo, no estudo coorte clínico EURODIALE $^{7}$ sobre fatores de prognóstico de doentes com pé diabético com efeitos avaliados através do OR, ou no estudo $\mathrm{EVAPE}^{8}$ sobre determinantes de mortalidade de causa vascular, também avaliados com recurso ao OR.

Last no dicionário de epidemiologia ${ }^{4}$ aponta, entre vários tipos de OR, para a existência de dois OR que na prática representam o mesmo resultado; o OR baseado nos odds da doença (com os odds a compararem doentes versus não doentes) num estudo coorte ou num estudo trans- versal de prevalência e o OR baseado na razão dos odds da exposição (com os odds a compararem expostos versus não expostos) num estudo caso-controlo. Em termos práticos, o OR determinado partindo da exposição para a doença num estudo coorte ou de prevalência, é exactamente o mesmo em valor numérico, que o OR determinado partindo da doença para a exposição num estudo caso-controlo. Facilmente se vê, e aplicando o exemplo da Talidomida em que se determinou o OR partindo da exposição para a doença, que se este fosse determinado partindo da doença para a exposição se obteria exactamente o mesmo valor numérico OR $=(41 / 1) /(5 / 300)=2460$. Este resultado foi pela primeira vez mostrado por Cornfield em $1951,{ }^{9}$ e veio demonstrar algo de muito importante para a investigação epidemiológica. Mais especificamente, o OR calculado num estudo caso controlo, ou seja o OR partindo da doença para a exposição (estudo caso-controlo), é exatamente igual ao OR partindo da exposição para a doença (estudo coorte), sendo este último aquele que mais interessa conhecer quando pretendemos medir a associação entre uma exposição e uma doença.

Apesar do OR ser uma medida epidemiológica de efeito tal como no exemplo da Talidomida, representando naturalmente a razão de riscos entre expostos e não expostos, vários autores apontam frequentemente que o OR pode sobrestimar o efeito em estudo, nomeadamente, para o caso de a doença em estudo não ser rara.,10-12 Considerando o nosso exemplo da Talidomida, o risco de malformação nos expostos era $41 / 46=89 \%$ e o risco de malformação nos não expostos seria $1 / 300=0,3 \%$. Consequentemente, a razão de riscos ou risco relativo (RR) seria avaliada em $\mathrm{RR}=89 \% / 0,3 \%=297$, ou seja, um valor bem inferior ao OR, embora em termos práticos com as mesmas conclusões catastróficas do OR.

Para o caso de uma doença ser rara, ou em termos práticos, cuja taxa de incidência ou de prevalência seja inferior a $10 \%$, o OR aproxima-se da razão de riscos entre expostos e não expostos. ${ }^{11}$ Sendo a doença ou o resultado de saúde em estudo mais frequente (> 10\%), então o OR sobrestima a razão de riscos. ${ }^{11,12}$

De modo a corrigir o viés do OR em estudos de doenças mais frequentes, Zhang e $\mathrm{Yu}^{13}$ propuseram uma correção bastante simples no OR de modo a obter o valor do RR e seu intervalo de confiança. No entanto, esta correção não se revelou um procedimento válido, em especial para o caso do OR necessitar de ajustamento para eventuais fatores de confundimento, ${ }^{11}$ digamos, através da usual técnica de regressão logística múltipla. ${ }^{14}$ 
Com objetivo de obter medidas de associação ajustadas para vários fatores de confundimento, outras técnicas estatísticas foram sugeridas, em substituição da análise de regressão logística, tal como a análise de regressão de Poisson, ${ }^{11}$ que permite obter estimativas mais válidas do RR ajustado para eventuais fatores de confundimento.

Neste artigo, pretendemos apresentar e discutir o viés introduzido ao usar o OR como estimativa do RR em função da frequência da doença ou resultado de saúde, assim como, as diferentes abordagens de análise para ajustar para confundimento o valor do OR e do RR. Discutiu-se ainda a validade do OR como estimador do RR de acordo com o desenho do estudo epidemiológico.

\section{MATERIAL E MÉTODOS}

Simulámos numa coorte de 200 indivíduos com 100 expostos a um hipotético fator de risco e 100 não expostos, dois diferentes cenários, um com a doença mais rara conduzindo a uma taxa de incidência global na amostra de $6,5 \%$, e outra com a doença mais frequente conduzindo a uma taxa de incidência de $65 \%$. Em ambas, os cenários simulados o RR determinado pela razão de taxas de incidência é igual, $R R=2,25$, indicando que o risco de doença nos expostos é 2,25 vezes o risco dos não expostos. Para esta simulação determinámos as estimativas brutas do OR e RR pela abordagem clássica e pela regressão logística e regressão Poisson respetivamente.

Seguidamente introduzimos para cada cenário uma eventual variável de confundimento. Aqui, determinámos o OR e o RR ajustados pela análise estratificada de Mantel-Haenszel ${ }^{10}$ e comparámo-los com o OR obtido por regressão logística e o RR obtido por regressão de Poisson. A regressão de Poisson foi efectuada considerando como unitário o tempo em risco para cada indivíduo. ${ }^{11}$

Considerámos o intervalo de confiança a 95\% para todas as medidas de efeito consideradas. Todos os cálculos foram efectuados em SPSS versão 20, com excepção do risco relativo da análise estratificada de Mantel-Hanszel em que foi utilizado o programa online OpenEpi versão 3.

\section{RESULTADOS}

A Tabela 2 apresenta os resultados da simulação que relaciona a exposição com a doença, tanto para o cenário em que a taxa de incidência é mais rara $(6,5 \%)$ como para o cenário com taxa de incidência mais frequente (65\%). Quando a taxa de incidência é mais rara, observa-se que a estimativa do OR está bem próxima da estimativa do RR estimado pela razão de taxas de incidência. No entanto, para a taxa de incidência de $65 \%$, o OR sobrestima claramente o mesmo valor de RR.

Podemos ainda verificar que as estimativas do OR e do RR obtidas, através dos métodos de regressão (Logística e Poisson) são, respetivamente equivalentes, às estimativas pontuais obtidas pela razão das odds e pela razão de taxas de incidência, quer o evento seja mais raro ou mais frequente.

Ainda um aspeto importante que se observa na Tabela 2, é que o intervalo de confiança para o RR determinado pela regressão de Poisson, é sempre mais largo que o intervalo de confiança da razão de taxas de incidência, independentemente do nível de raridade da incidência. $O$ mesmo não se verifica com o intervalo de confiança para o OR obtido pela regressão logística que é equivalente ao obtido pelo método clássico de aproximação de Wolf implementado no SPSS 20.0.

Ao considerar-se um eventual fator de confundimento para o cenário de taxa de incidência mais frequente (65\%), observa-se na análise estratificada que a razão de taxas de incidência baixa de $R R=2,25$ para $R R a=1,89$ após ajustamento - Tabela 3 - o que revela algum nível de confundimento. O ORa ajustado na regressão logística para o mesmo fator de confundimento continua a sobrestimar

Tabela 2 - OR e RR estimado para diferentes níveis de raridade da incidência

\begin{tabular}{|c|c|c|c|c|c|c|c|c|c|c|c|}
\hline $\mathrm{TI}=6,5 \%$ & Casos & TI & Total & OR & IC OR & $\begin{array}{c}\text { OR } \\
\text { Logit }\end{array}$ & $\begin{array}{l}\text { IC OR } \\
\text { Logit }\end{array}$ & $\mathbf{R R}$ & $\begin{array}{l}\text { IC } \\
\text { RR }\end{array}$ & $\begin{array}{c}\text { RR } \\
\text { Poisson }\end{array}$ & $\begin{array}{c}\text { IC RR } \\
\text { Poisson }\end{array}$ \\
\hline Exp & 9 & $9 \%$ & 100 & 2,37 & 0,71 & 2,37 & 0,71 & 2,25 & 0,72 & 2,25 & 0,69 \\
\hline N Exp & 4 & $4 \%$ & 100 & & 7,98 & & 7,98 & & 7,07 & & 7,31 \\
\hline $\mathrm{Tl}=65 \%$ & Casos & $\mathrm{TI}$ & Total & OR & IC OR & $\begin{array}{c}\text { OR } \\
\text { Logit }\end{array}$ & $\begin{array}{l}\text { IC OR } \\
\text { Logit }\end{array}$ & $\mathbf{R R}$ & $\begin{array}{l}\text { IC } \\
\text { RR }\end{array}$ & $\begin{array}{c}\text { RR } \\
\text { Poisson }\end{array}$ & $\begin{array}{c}\text { IC RR } \\
\text { Poisson }\end{array}$ \\
\hline Exp & 90 & $90 \%$ & 100 & 13,50 & 6,28 & 13,50 & 6,28 & 2,23 & 1,75 & 2,25 & 1,56 \\
\hline$N \exp$ & 40 & $40 \%$ & 100 & & 29,04 & & 29,04 & & 2,89 & & 3,27 \\
\hline
\end{tabular}

TI - Taxa de incidência; Exp - Expostos a factor de risco; OR - Odds Ratio; IC OR - Intervalo de confiança a 95\% para o OR; OR Logit - Odds Ratio estimado por regressão de logística; IC OR Logit - Intervalo de confiança a 95\% para o OR de logística ; RR - Risco Relativo estimado pela razão de taxs de incidência; IC RR - Intervalo de confiança a 95\% para o RR; RR Poisson - Risco Relativo estimado por regressão de Poisson; IC RR Poisson - Intervalo de confiança a 95\% para o RR de Poisson. 
claramente o RRa obtido na análise estratificada. No entanto em comparação com o seu recíproco bruto, baixa de $\mathrm{OR}=13,5$ para $\mathrm{ORa} \mathrm{MH}=10,63$, mostrando desta forma que, independentemente da medida de associação, esta variável de confundimento estaria a produzir um viés positivo. No entanto tanto o OR como o ORa estão a sobrestimar claramente o efeito em estudo.

Quando analisados os resultados do ajustamento obtido pelos métodos de regressão, verifica-se que o RRa e ORa obtidos respetivamente por regressão de Poisson e logística, aproximam-se dos seus equivalentes ORa e RRa obtido por análise estratificada, mantendo-se a sobrestimação dos odds ratios relativamente aos riscos relativos.

No cenário de doença rara $(\mathrm{TI}=6,5 \%)$ verifica-se igualmente uma variação entre os $\operatorname{RR}(2,25)$ e OR $(2,37)$ brutos e os seus respetivos ajustados pelo método de Mantel-Hanszel $(\operatorname{RRa}=3,19$ e Ora $=3,59)$. Neste caso, revelando que o efeito de confundimento estava a provocar um viés negativo. Mas é importante notar também que, ao contrário do observado no cenário da doença frequente, os valores do RR e OR ajustados pelos vários métodos apresentados são próximos entre si variando entre 3,19 e 4,03.

No entanto, e tal como se tinha referido na Tabela 2, também na situação do ajustamento do RR para uma variável de confundimento através da regressão de Poisson, se observam intervalos de confiança mais largos, em comparação com os intervalos de confiança da análise estratificada (Tabela 3).

\section{DISCUSSÃO}

Este estudo permitiu mostrar que em situações em que não é observado o pressuposto de doença rara, o OR sobrestima o RR. No entanto, a abordagem de análise de regressão de Poisson permitiu atingir estimativas do RR idênticas à razão de taxas de incidência e muito próximas do RR ajustado para confundimento através de análise estratificada.
Posto isto, levanta-se a óbvia questão acerca da validade de utilização do OR e consequentemente do seu procedimento de análise de regressão logística múltipla, para ajustar o OR a eventuais variáveis de confundimento, em estudos de incidência e de prevalência. Apesar das limitações envolvidas, pensamos que a utilização de regressão logística múltipla e consequentes OR ajustados devem ser considerados como uma abordagem válida de análise, embora com algumas precauções ao nível das conclusões, nomeadamente ao nível da interpretação do OR como RR ou como razão de prevalências (RP) em situação de doença mais frequente.

Em primeiro lugar devemos considerar que a análise de regressão logística múltipla é um procedimento muito popular no meio de análise de dados epidemiológicos. Verifica-se por exemplo que o livro 'Applied Logistic Regression' de Hosmer and Lemeshow ${ }^{14}$ é apresentado como o livro de bioestatística e epidemiologia mais citado ${ }^{15}$ na literatura científica epidemiológica desde a sua primeira edição em 1989, recebendo desde essa data uma média anual de 876,2 citações/ano. O número 2 deste ranking é ocupado pelo livro 'Statistical methods for rates and proportions' de Fleiss ${ }^{16}$ com 447,4 citações/ano, ou seja, aproximadamente metade das citações. Este resultado demonstra a enorme popularidade e utilização desta técnica estatística de regressão logística na investigação epidemiológica internacional. Neste sentido, e na prática habitual de investigação epidemiológica, é quase inconcebível pensar num estudo em que pretendemos determinar fatores associados a uma prevalência, sem pensar em efectuar uma regressão logística múltipla para sintetizar os fatores mais relevantes. Para além deste aspeto da popularidade do OR, devemos pensar que num estudo caso-controlo, a abordagem mais válida para análise e estimação do RR é o OR e consequente regressão logística múltipla. No entanto, na situação mais usual em que os controlos são selecionados entre os indivíduos livres da doença no final do período de seleção dos

Tabela 3 - RR e OR ajustado para um factor de confundimento

\begin{tabular}{|c|c|c|c|c|c|c|c|}
\hline \multicolumn{8}{|c|}{$\mathrm{TI}=6,5 \%$} \\
\hline RRa MH & IC RRa MH & RRa Poisson & IC RRa Poisson & ORa MH & IC ORa MH & ORa Logit & IC ORa Logit \\
\hline \multirow[t]{2}{*}{3,19} & 1,10 & 3,43 & 1,04 & 3,59 & 1,06 & 4,03 & 1,14 \\
\hline & 9,23 & & 11,28 & & 10,88 & & 14,29 \\
\hline \multicolumn{8}{|c|}{$\mathrm{Tl}=65 \%$} \\
\hline RRa MH & IC RRa MH & RRa Poisson & IC RRa Poisson & ORa MH & IC ORa MH & ORa Logit & IC ORa Logit \\
\hline \multirow[t]{2}{*}{1,89} & 1,52 & 1,97 & 1,33 & 10,63 & 4,83 & 10,81 & 4,87 \\
\hline & 2,36 & & 2,90 & & 23,43 & & 23,99 \\
\hline
\end{tabular}

RRa MH - RR ajustado por análise estratificada de Mantel-Haenszel; ORa MH - OR ajustado por análise estratificada de Mantel-Haenszel; IC - Intervalo de confiança a 95\%; ORa OR ajustado por regressão logística; RRa Poisson - RR ajustado por regressão de Poisson 
casos, o OR irá sobrestimar o RR se a doença não for rara Mas, este facto não é regra nos estudos caso-controlo, pois existem determinados delineamentos, tal como o estudo caso-coorte e o caso-controlo com seleção dos controlos emparelhados no tempo pelos casos incidentes. ${ }^{4}$ Nestas situações de estudos caso-controlo, o OR obtido é respetivamente um estimador não enviesado do $\mathrm{RR}$ e da razão de taxas de incidência independentemente da frequência da doença. ${ }^{17}$

Para além disto, muitos dos estudos epidemiológicos podem ser considerados exploratórios como o EURODIA$\mathrm{LE}^{7}$ e $\circ \mathrm{EVAPE}^{8}$ em que se pretendem explorar múltiplos fatores associados ao resultado de saúde de interesse. Em situações de investigação desta natureza, digamos mais exploratória, a análise de regressão logística múltipla é uma boa ferramenta para ajudar a identificar os fatores mais importantes. Os OR seleccionados por análise de regressão logística múltipla orientam claramente sobre o sentido da associação encontrada (positiva versus negativa). O único cuidado a ter, e em especial para o caso da doença ou resultado de saúde não ser raro, está na interpretação do OR, ou seja, deve-se ter cautela para não interpretar o OR diretamente como um RR ou um Benefício Relativo (BR) em estudos prospectivos, ou como uma razão de prevalências (RP) num estudo transversal. No entanto, pode-se perfeitamente interpretar o OR acerca do sentido da associação, ou seja, pode-se concluir se a exposição está associado a mais doença - associação positiva - ou se a exposição está associado a menos doença - associação negativa.

Quando o resultado de saúde não for raro e para o caso de um estudo prospetivo pretender investigar uma exposição concreta sujeita a confundimento, por exemplo, num estudo experimental comparativo ou num estudo em que se pretendem por hipótese comparar uma coorte específia de expostos versus outra de não expostos, então nestas situações pensamos que é naturalmente mais válido utilizar análise estratificada para estimar o RR ou o BR, caso exista uma única variável de confundimento com categorias que permitam a estratificação, ou a análise de regressão múltipla de Poisson caso existam diversas variáveis de confundimento. Se num estudo transversal existir o objectivo concreto de estimar uma razão de prevalências ajustada para diversos fatores de confundimento, então a análise de regressão de Poisson também poderá ser um procedimento mais válido que a análise de regressão logística.

A análise de regressão de Poisson existe nos programas estatísticos mais conhecidos em epidemiologia, como o SPSS, o STATA e o SAS. Este método de análise é equivalente à análise de regressão de $\operatorname{Cox}^{11}$ com tempo individual unitário, embora este último tipo de análise seja mais

\section{REFERÊNCIAS}

1. McBride WG. Thalidomide and Congenital Abnormalities. Lancet. 1961;2:1958.

2. Mellin GW, Katzenstein M. The saga of Thalidomide: neuropathy to embryopathy, with case reports of congenital abnormalities. N Engl J Med. 1962;267:1238-43. popular quando aplicado na análise de sobrevivência.

Apesar da vantagem da análise de regressão de Poisson permitir estimativas mais válidas para a razão de riscos em estudos prospetivos ou razão de prevalências em estudos transversais, uma limitação deste método ficou evidente na simulação efetuada, ou seja, os intervalos de confiança são mais largos em comparação com métodos padrão como o intervalo de confiança para a razão de riscos, ou o intervalo de confiança para a razão de riscos ajustada para confundimento na análise estratificada. Esta evidência já tinha sido observada na literatura. ${ }^{11}$ Estes intervalos de confiança mais largos podem aumentar o erro tipo II dos testes de hipóteses tornando-os conservadores, com a consequência de não ser encontrado um efeito estatisticamente significativo que de fato existe. Esta limitação da análise de regressão de Poisson aplicada neste contexto deve ser tomada em atenção e em especial para as situações de investigação com amostras mais pequenas. Em substituição da regressão de Poisson, também podem ser sugeridos outros métodos tal como a regressão Log-Binomial, ${ }^{11}$ embora também apresentem limitações.

\section{CONCLUSÃO}

Em conclusão, consideramos que o OR e a regressão logística múltipla devem ser usados em qualquer estudo caso-controlo e em estudos exploratórios prospetivos e transversais de múltiplos determinantes ou fatores associados à doença ou resultado de saúde em estudo. Em estudos prospectivos e transversais, o OR não deve ser interpretado diretamente como um RR, BR ou RP caso o resultado de saúde não seja raro, embora se possam tirar conclusões acerca do sentido da associação. Em situação de resultado de saúde mais frequente, caso se deseje estimar um específico RR, BR ou RP ajustado para diversos fatores de confundimento, a análise de regressão múltipla de Poisson é uma alternativa válida à regressão logística múltipla.

\section{AGRADECIMENTOS}

Isabel Andrade da Escola Nacional de Saúde Pública e Luís Veloso, Filipa Negreiro Eurotrials Consultores Científicos.

\section{CONFLITO DE INTERESSES}

Os autores declaram que não houve conflito de interesses na realização deste trabalho.

\section{FONTES DE FINANCIAMENTO}

Os autores declaram a inexistência de fontes de financiamento externas.

3. Doll R, Peto R, Boreham J, Sutherland I. Mortality in relation to smoking: 50 years observations on male British doctors. BMJ. 2004;328:1519.

4. Last JM. A dictionary of epidemiology. $3^{\text {rd }}$ ed. New York: Oxford University Press;1995.

5. Rothman KJ, Greenland S. Modern epidemiology. $2^{\text {nd }}$ ed. Philadelphia: 
Lippincott Williams \& Wilkins; 1998.

6. Gordis L. Epidemiology. $3^{\text {rd }}$ ed. Philadelphia: Elsevier Saunders; 2004.

7. Prompers L, Schaper N, Apelqvist J, Edmonds M, Jude E, Mauricio D, et al. Prediction of outcome in individuals with diabetic foot ulcers: focus on the differences between individuals with and without peripheral arterial disease. Diabetologia. 2008;51:747-55.

8. Carraça I, Amorim P, Ferreira G, Legrant C, Pinto M. Estudo EVAPE (Exploring Vascular Protective Effects). Rev Port Clin Geral. 2009;29:15767.

9. Cornfield J. A method for estimating comparative rates from clinical data Applications to cancer of the lung, breast, and cervix. J Natl Cancer Inst. 1951;11:1269-75.

10. Egger M, Smith GD, Altman D. Systematic reviews in health care. Metaanalysis in context. London: BMJ Books; 2001.

11. McNutt $L$, Wu $C$, Xue $X$, Hafner JP. Estimating the relative risk in co- hort studies and clinical trials of common outcomes. Am J Epidemiol. 2003;157:940-3.

12. Nurminen M. To use or not to use the odds ratio in epidemiological analysis? Eur J Epidemiol. 1995;11:365-71.

13. Zhang J, Yu KF. What's relative risk? A method of correcting the odds ratio in cohort studies of common outcomes. JAMA. 1998;280:1690-1.

14. Hosmer DW, Lemeshow S. Applied logistic regression. New York: John Wiley \& Sons; 1989

15. Porta M, Vandenbroucke JP, loannidis JP, Sanz S, Fernandez E, Bhopal $R$, et al. Trends in citations to books on epidemiological and statistical methods in the biomedical literature. PLoS One. 2013;8:e61837.

16. Fleiss JL. Statistical methods for rates and proportions. New York: Wiley; 1973.

17. Pearce N. What does the odds ratio estimate in a case-control study? In J Epidemiol. 1993;22:1189-92. 